\title{
Factors associated with survival in dogs with a histopathological diagnosis of hepatocellular carcinoma: 94 cases (2007-2018)
}

\author{
James Moyer, Daniel J. Lopez, Cheryl E. Balkman and Julia P. Sumner* \\ Department of Clinical Sciences, College of Veterinary Medicine, Cornell University, Ithaca, NY, 14850, USA
}

\begin{abstract}
Background: Hepatocellular carcinoma (HCC) is the most common form of primary liver cancer in dogs. Despite this, relatively few reports of this disease exist pertaining to prognostic factors and outcome.

Aim: To evaluate factors associated with survival in dogs with all subtypes of HCC diagnosed on histopathology.

Methods: A retrospective single institutional study was carried out on 94 client-owned dogs with a histopathologic diagnosis of HCC between 2007 and 2018 obtained by biopsy (21/94) or attempted definitive resection (73/94). Signalment, preoperative features, surgical findings, and postoperative outcomes were recorded. Associations between survival to discharge data were collected and univariable logistical regression was carried out. Kaplan-Meier survival analysis was carried out to identify negative risk factors for long-term prognosis.

Results: The median survival time (MST) for all patients was 707 days $(95 \% \mathrm{CI}=551-842)$. MST was not significantly different $(p>0.05)$ between patients who had suspected versus incidentally diagnosed HCC (695 vs. 775 days), between complete versus incomplete surgical margins (668 vs. 834 days), or between patients with massive subtype versus nodular/diffuse subtype (707 vs. 747 days). Logistical regression identified an association with the excision of the right medial lobe and risk of perioperative death $(\mathrm{OR}=9.2$, CI 1.5-55.9, $p=0.016)$. An American Society of Anesthesiologists score $\geq 4$, disease present within the quadrate lobe, and elevated blood urea nitrogen, potassium or gamma-glutamyltransferase were identified as negative prognosticators during multivariable Cox regression. Preoperative imaging (ultrasound or CT) agreed with the surgical location in $91 \%$ of the cases. Preoperative cytology was consistent with a diagnosis of HCC in 15/32 (46.9\%) cases.

Conclusion: Type of diagnosis (incidental vs presumed), completeness of excision, and subtype were not associated with MST in this study. Preoperative identification of tumors within the central division may be related to a less favorable outcome. Results of preoperative cytology were not highly sensitive for identifying a malignancy.

Keywords: Cancer, Canine, Hepatocellular carcinoma, Liver, Survival analysis.
\end{abstract}

\section{Introduction}

Primary hepatobiliary cancer is uncommon in the dog, accounting for approximately $1 \%$ of all canine neoplasia found on necropsy (Patnaik et al., 1980). These neoplasms most commonly arise from hepatocytes, but biliary, neuroendocrine, and stromal cell tumors have all been recognized (Balkman, 2009). By far, the most common form of primary liver cancer is hepatocellular carcinoma (HCC), accounting for approximately 50\%$77 \%$ of primary hepatobiliary cases observed (Patnaik et al., 1980; Van Sprundel et al., 2013). Despite being the most common liver tumor seen in the dog, relatively few reports of this disease exist in the literature in regard to prognostic factors and outcome.

HCC has three gross morphological subtypes: massive, nodular, and diffuse (Patnaik et al., 1980). These subtypes have previously been defined as massive generally describing a singular large mass, the nodular form representing nodules of differing sizes and the diffuse type representing diffusion of the neoplasm throughout the liver (Patnaik et al., 1980). The prognosis between these subtypes may differ (Patnaik et al., 1981; Liptak and Withrow, 2013); however, the association between subtype and survival has not been extensively studied. It is generally accepted that dogs with the massive form have the best prognosis (Liptak and Withrow, 2013); likely because the massive form is often seen to be amenable to surgical treatment. Surgical intervention for patients with massive HCC $(n=42)$ has been associated with prolonged survival times ( $>1,460$ days) when compared to conservative treatment (270 days), but the sample size for the conservative treatment group $(n=6)$ in this particular study was relatively small (Liptak et al., 2004). Surgical intervention for massive $\mathrm{HCC}$ via partial or complete liver lobectomy has historically been the treatment recommendation of choice particularly as recurrence or local metastasis appears to be rare (Kosovsky, 1989; Liptak et al., 2004; Kinsey et al., 2015; Matsuyama et al., 2017). To date, there have been conflicting reports in regard to the connection between complete surgical resection of $\mathrm{HCC}$ and prolonged median survival time (MST). In a study of $37 \mathrm{dogs}$, the resection of massive HCC with clean surgical margins resulted in

*Corresponding Author: Julia Sumner. Department of Clinical Sciences, College of Veterinary Medicine, Cornell University, 
significantly longer survival times $(>1,836$ days $)$ versus those with incomplete margins (756 days) (Matsuyama et al., 2017). This is in contrast to other reports where no association was found between complete surgical excision and MST (Liptak et al., 2004; Linden et al., 2019).

Several studies have identified an association with HCC tumor location and outcome (Liptak et al., 2004; Matsuyama et al., 2017; Linden et al., 2019). MST for HCC of the left division (>1,460 days) is reportedly higher than that affecting the central (>795 days) and right division (365 days) (Liptak et al., 2004). This difference is likely due in part to the technical challenges associated with surgery in the right and central division, leading to higher perioperative deaths. Intraoperative mortality approached $40 \%$ with the resection of right-sided tumors in one study (Liptak et al., 2004). Similarly, dogs with surgically addressed hepatic neoplasia confined to the central division have increased complications, in particular hemorrhage, when compared to those with left-sided disease (Linden et al., 2019). It is therefore important to be able to accurately identify tumor location prior to surgery in an effort to guide owner expectations and prepare for complications that may arise.

The current literature on canine HCC is limited and has only identified risk factors in a small sample of patients with the massive form, leaving a void of information regarding survival times among the different subtypes of HCC. Additionally, little is known about the accuracy of preoperative diagnostics which are essential for surgical planning and to guide owner expectations. The objective of this study was to identify associations with both short-and long-term survival in dogs with HCC, with specific regard to histological subtype, surgical margins, and whether or not definitive surgical removal was attempted. Due to the seeming low metastatic rate of HCC, we hypothesized that the completeness of surgical margins would not significantly alter patient MST and that dogs with attempted surgical removal would have longer MST when compared to those that only underwent a biopsy. In addition, we evaluated the accuracy of preoperative testing including cytology, ultrasound, and computed tomography (CT) in the diagnosis and determination of tumor location prior to surgery. We hypothesized that CT would be more accurate than ultrasound when identifying preoperative location, and that cytology would be a useful preoperative diagnostic in differentiating $\mathrm{HCC}$ from other processes.

\section{Case identification \\ Materials and Methods}

Records from the Animal Health Diagnostic Center at the Cornell University College of Veterinary Medicine were searched for canine liver biopsies with a histologic diagnosis of HCC from June 2007 to September 2018. Histological samples were obtained surgically from patients at the Cornell University Hospital for Animals for the purpose of either definitive treatment of a liver tumor or for diagnostic biopsy. Cases were excluded if subsequent examination of histopathological samples amended the diagnosis to something other than HCC, if there was a secondary neoplastic process within the patient, if the medical record was significantly incomplete or unavailable, or survival data were not obtained by May 24, 2019.

\section{Data acquisition}

Based on the results of the histopathological query, electronic records of each patient were evaluated for completeness, and data were obtained. Preoperative variables included information on signalment (breed, sex, neuter status, weight, and age), preoperative blood work (serum chemistry, complete blood count, prothrombin time, and partial thromboplastin time), preoperative imaging (ultrasound and CT), and cytology obtained via fine needle aspirate (FNA). Surgical variables included information on anesthesia American Society of Anesthesiologists (ASA) score, presence of hypotension defined as a mean arterial blood pressure $<60 \mathrm{mmHg}$, need for administration of a blood transfusion or colloids), whether definitive surgical resection was attempted, evidence of gross metastasis on abdominal exploration, and the incidence of intraoperative death or euthanasia. Gross tumor characteristics included tumor location, involvement of multiple lobes, maximal tumor diameter recorded at surgery, and morphological subtype. Postsurgical data collected included the administration of any adjuvant therapy (radiation or chemotherapy) whether the patient was alive or date and cause of death.

Attempted definitive surgical resection was defined as cases that underwent planned surgical resection of a mass for local control or resolution of HCC. Surgical biopsies were obtained either from dogs where the tumor was deemed unresectable at the time of surgery, or from dogs undergoing celiotomy for other disease processes and incidental gross hepatic lesions were noted intraoperatively. Tumor location was classified as either right division (right lateral lobe, caudate lobe), central division (right medial lobe, quadrate lobe), or left division (left medial lobe, left lateral lobe) as previously defined (Sleight and Thomford, 1970).

Cytological agreement was defined as a complete agreement when a definitive diagnosis of $\mathrm{HCC}$ was made, as partial agreement when a malignant diagnosis was made and/or HCC was listed as a differential, and in disagreement when the sample was non-diagnostic, or an alternative non-malignant diagnosis was made. Imaging agreement was defined as the ability to correctly localize a lesion to the left, central, or right liver division without failing to identify other liver pathologies. Disagreement was defined as either an inability to identify the side involved, incorrect identification of the lesion location, or failing to 
identify lesions in other liver lobes. Survival time was defined as the date of surgery until the date of death. Date of death was acquired either from the medical record, from direct communication with the owner for this study, or direct communication with the primary veterinarian for this study.

\section{Statistical analysis}

Continuous data were assessed for normality using the Shapiro-Wilk test. Normal data were reported as mean ( \pm standard deviation) and non-normal data as median (interquartile range). Continuous biochemical variables were compared between incidental versus definitive surgical treatment using a Wilcoxon rank sum test. Simple univariable logistical regression was used to identify variables associated with death prior to discharge for patients undergoing attempted definitive surgical treatment. Variables were reported if the likelihood ratio $p$-value was $<0.05$. Values with perfect prediction were reported separately. Univariable Cox regression estimates were carried out evaluating survival for all factors and Kaplan-Meier survival curves were generated for factors of interest. Hazard ratios, 95\% confidence intervals, and $p$-values were reported. Survival curves were right-sided censored if patients remained alive at the time of statistical analysis or were known to have died from a cause unrelated to HCC. Patients remained uncensored if their death was confirmed to be related to the diagnosis of HCC or if the cause of death was undetermined. Multivariable Cox regression was carried out to create a model evaluating overall survival for all patients and patients undergoing definitive surgical resection. Variables were reported and included within the model if their likelihood ratio test $p$-value was $<0.10$. Backward elimination was carried out until all variables retained a likelihood ratio test $p$-value $\leq 0.05$. All variables within the final model were evaluated for interactions and included within the final model if significant. Proportional hazard assumptions were evaluated with Shoenfeld residuals by using the score test, with $p$-value $>0.05$ indicating lack of violation of the proportionality assumption. All statistical calculations were carried out using commercial software (Stata, V 15.1, College Station, TX).

\section{Ethical approval}

This study was carried out by collecting data on clientowned animals undergoing treatment for naturally occurring disease. Informed consent to use data for research purposes was obtained from each owner.

\section{Results}

\section{Signalment, preoperative, and anesthetic data}

Following query of the histopathological data bank, 131 biopsies were identified with a diagnosis of HCC. After application of exclusion criteria, 94 unique patients were identified having underwent either a surgical biopsy $(n=21)$ or attempted definitive surgical removal $(n=73)$ of HCC. Of the 21 patients undergoing surgical biopsy, 17 patients were diagnosed incidentally with $\mathrm{HCC}$ and 4 patients underwent biopsy of a tumor deemed unresectable by the operating surgeon. No patients who underwent surgical biopsy subsequently underwent attempted definitive surgical removal.

Of the dogs included, 52/94 (55.3\%) were male and $42 / 94(44.7 \%)$ were female. Three of the male patients were intact. None of the female patients were intact. Thirty-five breeds were identified, with the highest prevalence being either mixed breed (30/94, 31.9\%), golden retrievers $(11 / 94,11.7 \%)$, or Siberian huskies $(5 / 94,5.3 \%)$. The mean age at the time of the surgical event was 10.9 years ( \pm 2.43 months). The mean weight at the time of the surgical event was $21.6 \mathrm{~kg}( \pm 1.14 \mathrm{~kg})$. Complete blood counts and biochemical profiles were available for 93 of the 94 cases. Patients were classified as being anemic in $35(37.6 \%)$ cases, having thrombocytosis in $30(32.3 \%)$, being thrombocytopenic in 12/93 (12.9\%), having leukocytosis in 30/93 (32.3\%), or being leukopenic in $3 / 93(3.2 \%)$ cases. Patients with incidentally diagnosed HCC had lower alanine aminotransaminase (ALT) values [106 (65-223) U/L] (reference range $[\mathrm{RR}]=20-98 \mathrm{U} / \mathrm{l}$ ) and lower albumin values $[3.3(2-3.9) \mathrm{g} / \mathrm{dl}](\mathrm{RR}=3.1-4.2 \mathrm{~g} / \mathrm{dl})$ than patients with suspected HCC [428.5 (200-1,199.5) $\mathrm{U} / 1](p<0.001),[3.7(3.3-4.1) \mathrm{g} / \mathrm{dl}](p=0.019)$, respectively. Coagulation profiles were available for 48 patients. The mean PT time was $13.4( \pm 0.17)$ seconds $(\mathrm{RR}=11.0-15.5$ seconds $)$. The median activated partial thromboplastin time (aPTT) was 14.3 seconds (13.115.8) $(\mathrm{RR}=8.5-15.5$ seconds $)$. Prolonged coagulation times were noted in 2/48 PT readings and 5/48 aPTT readings.

Complete anesthetic records were available for 88/94 patients. Prior to anesthesia, the median assigned ASA score was 3 (2-4). Intraoperative hypotension occurred in 50/88 (56.8\%) patients. Intraoperatively and postoperatively, $8 / 88(9.0 \%)$ patients required blood transfusions and 16/88 (18.1\%) patients required colloids. The administration of blood products was not associated with a specific localization of disease $(p=$ 0.808).

Agreement between preoperative diagnostics and postoperative results

Prior to surgical intervention, 91 patients had preoperative imaging carried out. Of these imaging exams, 84 were evaluated with ultrasound and 41 were evaluated with CT. When considering the location of the tumor (left, central or right division), and whether metastasis was present, ultrasound agreed with surgical findings in 76/84 (90.5\%) of patients and CT agreed with surgical findings in 38/42 (90.5\%) patients. When both studies were available, the agreement between ultrasound and CT occurred in 32/35 (91.4\%) cases.

Preoperative cytology was carried out on 32 patients by FNA. Complete agreement between cytology and histopathology defined as a cytological diagnosis of HCC occurred in 15/32 (46.9\%) cases. Partial agreement 
with histopathology with a diagnosis of a malignancy of uncertain origin occurred in 4/32 (12.5\%) cases. Cytology attained a different (non-malignant) or nondiagnosis in 13/32 (40.6\%) cases.

\section{Surgical findings}

Of the 94 cases, 21 patients had a surgical biopsy and 73 patients underwent attempted definitive surgical removal of HCC. Of the 21 patients with surgical biopsies, four had biopsies taken after the tumor was deemed inoperable at the time of surgery. The remaining 17 cases had biopsies taken at the time of surgical management of other disease processes due to gross lesions noted on abdominal exploratory surgery. When definitive surgical resection was attempted, complete margins were achieved in $24 / 73$ patients. No patients were noted to have gross metastatic disease at the time of surgical intervention.

Multiple liver lobes were affected in 35/94 cases based on histopathological and surgical examination. In patients with only one lobe affected, the left lateral lobe was affected in 26/59 (44.1\%) cases, left medial lobe in $10 / 59(16.9 \%)$, right medial lobe in $10 / 59$ (16.9\%), quadrate lobe in 5/59 (8.5\%), right lateral lobe in $4 / 59(6.8 \%)$, and the caudate lobe in $4 / 59(6.8 \%)$. The median maximum tumor diameter, available in 77 patients, was $9 \mathrm{~cm}(6-14 \mathrm{~cm})$.

\section{Tumor morphology}

Tumors were classified as either massive $(n=$ $64)$, nodular $(n=21)$, or diffuse $(n=9)$. Of the 64 massive tumors, two $(3.1 \%)$ were considered poorly differentiated and four $(6.3 \%)$ were noted to have vascular invasion on histopathology

\section{Patient outcomes}

MSTs are shown in Figure 1. The MST for all patients from the time of surgery was 707 days $(\mathrm{CI}=551-842)$ (Fig. 2). Twelve out of 94 patients were still alive at the completion of the study, with one patient with a tumor that was deemed inoperable at surgery, remaining alive at 1,751 days. No patients had intraoperative death or were euthanized intraoperatively. No patients in this study underwent adjuvant radiation or chemotherapy for HCC. The cause of death was either related to death prior to discharge $(6 / 82)$, metastatic disease $(1 / 82)$, unrelated $(15 / 82)$, unknown $(55 / 82)$, or progressive liver disease $(5 / 82)$. All six dogs that died prior to discharge were undergoing surgery for attempted resection of tumors. No patients undergoing biopsy died prior to discharge.

Univariable logistical regression evaluating associations between patients undergoing attempted definitive surgical removal and death prior to discharge

Univariable logistical regression identified a significant association with the presence of disease within the right medial liver lobe and survival to discharge $(\mathrm{OR}=9.2, \mathrm{CI}$ $=1.5-55.9, p=0.016$ ) in patients undergoing attempted definitive surgical removal. Logistical regression could not be evaluated in patients having a leukocytosis, leukopenia, disease within the caudate lobe, a prolonged PT value, and presence of undifferentiated tumors due to all patients with prementioned variable surviving until

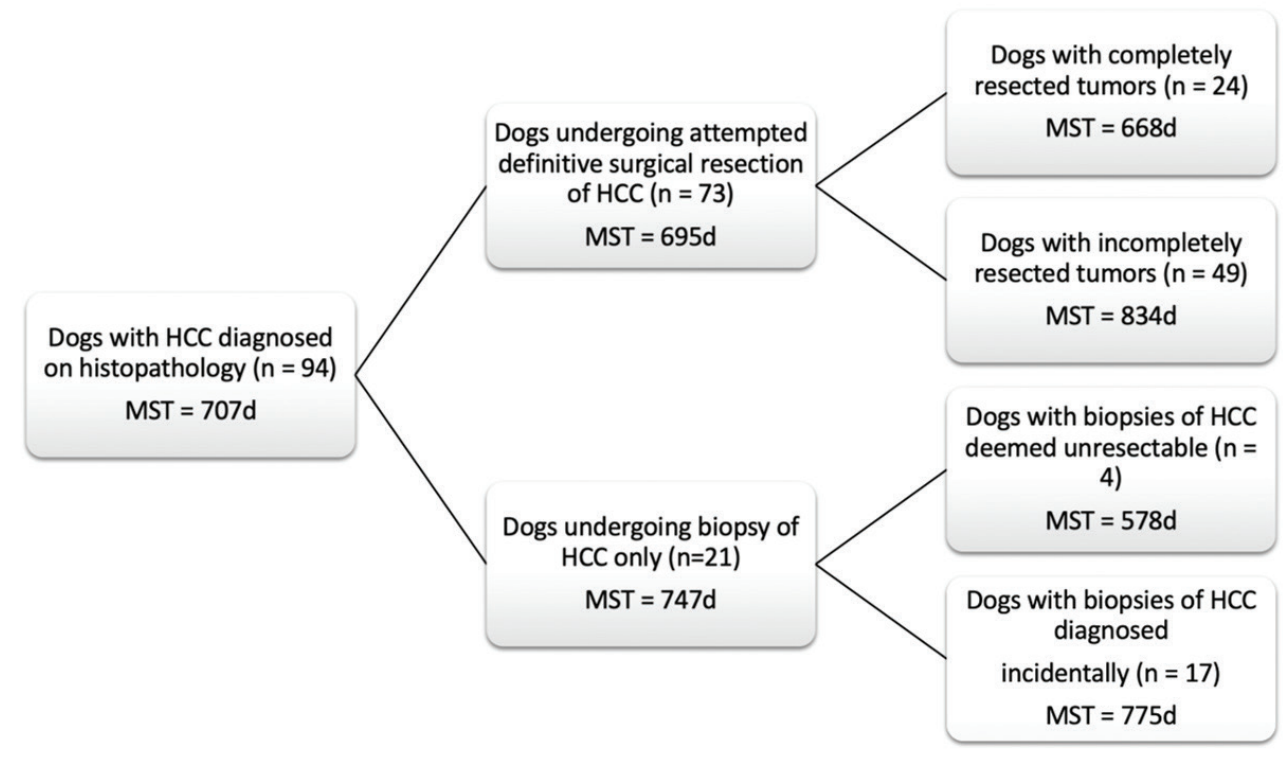

Fig. 1. Schematic demonstrating the MST of dogs following a histopathologic diagnosis of HCC. There was no significant difference in MST between those dogs undergoing definitive surgical resection and those undergoing biopsy. For those dogs undergoing definitive surgical resection, there was no significant difference in MST between those that had complete and incomplete surgical margins. Likewise, there was no significant difference in MST between dogs that had biopsies of HCC that was deemed unresectable at surgery, and those that had HCC diagnosed incidentally. 


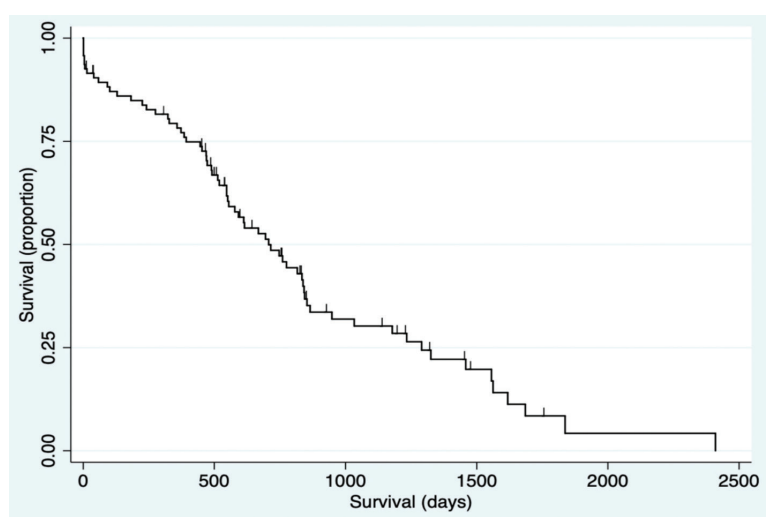

Fig. 2. Kaplan-Meier survival curve of the study population with a diagnosis of HCC.

discharge. In dogs with an attempted surgical removal of their tumors, no significant association was identified between survival to discharge and age, gender, weight, presence of anemia, presence of thrombocytosis/ thrombocytopenia, sodium, chloride, glucose, blood urea nitrogen (BUN), total bilirubin, total protein, albumin, ALT, alkaline phosphatase (ALP), gammaglutamyl transferase (GGT), cholesterol, bicarbonate, prolongation of aPTT, need for blood transfusion, need for colloid administration, presence of multiple lobes affected, presence of HCC in any lobe other than the right medial liver lobe, surgical margin, size of the tumor, subtype of the tumor, or whether the mass had invaded the vasculature on histopathology.

\section{Survival analysis for long-term outcome}

Survival curves were generated and evaluated to assess the effect of variables on long-term outcome (Fig. 3a-f). There was no significant difference identified in survival curves for patients undergoing definitive surgical resection for $\mathrm{HCC}(\mathrm{MST}=695$ days, $\mathrm{CI}=$ 519-844) versus biopsy (MST $=747$ days, $\mathrm{CI}=470$ 948) $(\mathrm{HR}=1.07, \mathrm{CI}=0.60-1.91, p=0.810)$ (Fig. 3a). There was no significant difference in MST for those dogs with incidentally diagnosed via a surgical biopsy during abdominal exploratory surgery for another disease process (MST $=775, \mathrm{CI}=241-948)$ and dogs with presumptive preoperative diagnosis of HCC [dogs with attempted surgical resection of a liver mass $(n=$ 73 ) or biopsy of a mass that was deemed unresectable $(n=4)](\mathrm{MST}=695$ days, $\mathrm{CI}=551-842)(\mathrm{HR}=0.98$, $\mathrm{CI}=0.52-1.84, p=0.943$ ) (Fig. 3b). For patients undergoing definitive surgical resection, there was no significant difference in survival curves for patients with complete $(\mathrm{MST}=668$ days, $\mathrm{CI}=446-838)$ versus incomplete margins (MST $=834$ days, $\mathrm{CI}=513-1,290)$ $(\mathrm{HR}=1.63, \mathrm{CI}=0.89-2.98, p=0.111)($ Fig. 3c). When disease was confined to a single lobe in patients undergoing definitive surgical removal, no significant difference was identified between patients with tumors in the right $(n=7, \mathrm{MST}=1,290$ days, CI $=834-\infty)$ versus central $(n=13, \mathrm{MST}=592$ days, $\mathrm{CI}=373-853)$
$(\mathrm{HR}=2.98, \mathrm{CI}=0.92-9.63, p=0.068)$, right versus left $(\mathrm{MST}=715$ days, $\mathrm{CI}=453-1,233)(\mathrm{HR}=2.09, \mathrm{CI}$ $=0.72-6.11, p=0.177)$, or central versus left division $(\mathrm{HR}=0.70, \mathrm{CI}=0.33-1.47, p=0.349)$ (Fig. 3d). No significant difference was found in long-term survival between patients with a massive subtype (MST $=707$ days, CI = 519-844) and those with either the diffuse or nodular subtypes (MST $=747$ days, $\mathrm{CI}=489-865)$ $(\mathrm{HR}=0.92, \mathrm{CI}=0.53-1.61, p=0.773)$ (Fig. 3e). There was no significant difference in survival between dogs that had disease in multiple lobes $(\mathrm{MST}=578, \mathrm{CI}=$ 470-816 days) versus dogs that had disease confined to a single lobe $(\mathrm{MST}=834$ days, $\mathrm{CI}=551=1,178)(\mathrm{HR}$ $=1.65, \mathrm{CI}=0.96-2.83, p=0.070)$ (Fig. 3f).

Results of multivariable Cox regression for all patients and the subgroup of patients undergoing attempted definitive surgical resection are presented in Tables 1 and 2. The final multivariable model for all patients retained disease within the quadrate lobe, an elevated potassium level, an elevated BUN level, and elevated GGT level, and being older as significantly impacting overall survival. No confounding interactions were identified. This model did not violate the proportionality assumption $(p=0.896)$. The final model for patients undergoing attempted definitive surgical resection retained an ASA score greater than or equal to 4, disease within the quadrate lobe, an elevated potassium, an elevated BUN value, and an elevated GGT score as significantly impacting survival. No confounding interactions were identified. This model did not violate the proportionality assumption $(p=0.879)$. Based on these models, Kaplan-Meier survival analysis (unadjusted) was carried out for all patients with ASA scores 4 or 5 versus 2 or 3 (Fig. 4) and patients undergoing attempted definitive surgical resection with disease present within the quadrate lobe versus not (Fig. $5)$. The MST of patients having an ASA score of 4 or 5 was 393 days $(\mathrm{CI}=14-612)$ compared to 775 days (CI $=578-853$ ) for patients with an ASA score of 2 or 3 . The MST of patients undergoing definitive surgery having disease present within the quadrate lobe was 489 days $(\mathrm{CI}=4-816)$ compared to 834 days $(\mathrm{CI}=551-1,178)$ for patients have disease absent in the quadrate lobe.

\section{Discussion}

The primary objective of this study was to carry out a survival analysis on a large cohort of dogs with all subtypes of HCC diagnosed on histopathology. The MST in this study was 707 days which is appreciably less than what has previously been reported in dogs with massive $\mathrm{HCC}$ ( $>1,460$ days) (Liptak et al., 2004). In contrast to previous reports, this study population included all subtypes of HCC. In addition, only a small percentage of our patients were alive at the conclusion of the study (around $12 \%$ ), minimizing right censoring of the population and providing a unique perspective on MST in dogs with all morphological forms of HCC. Despite these 

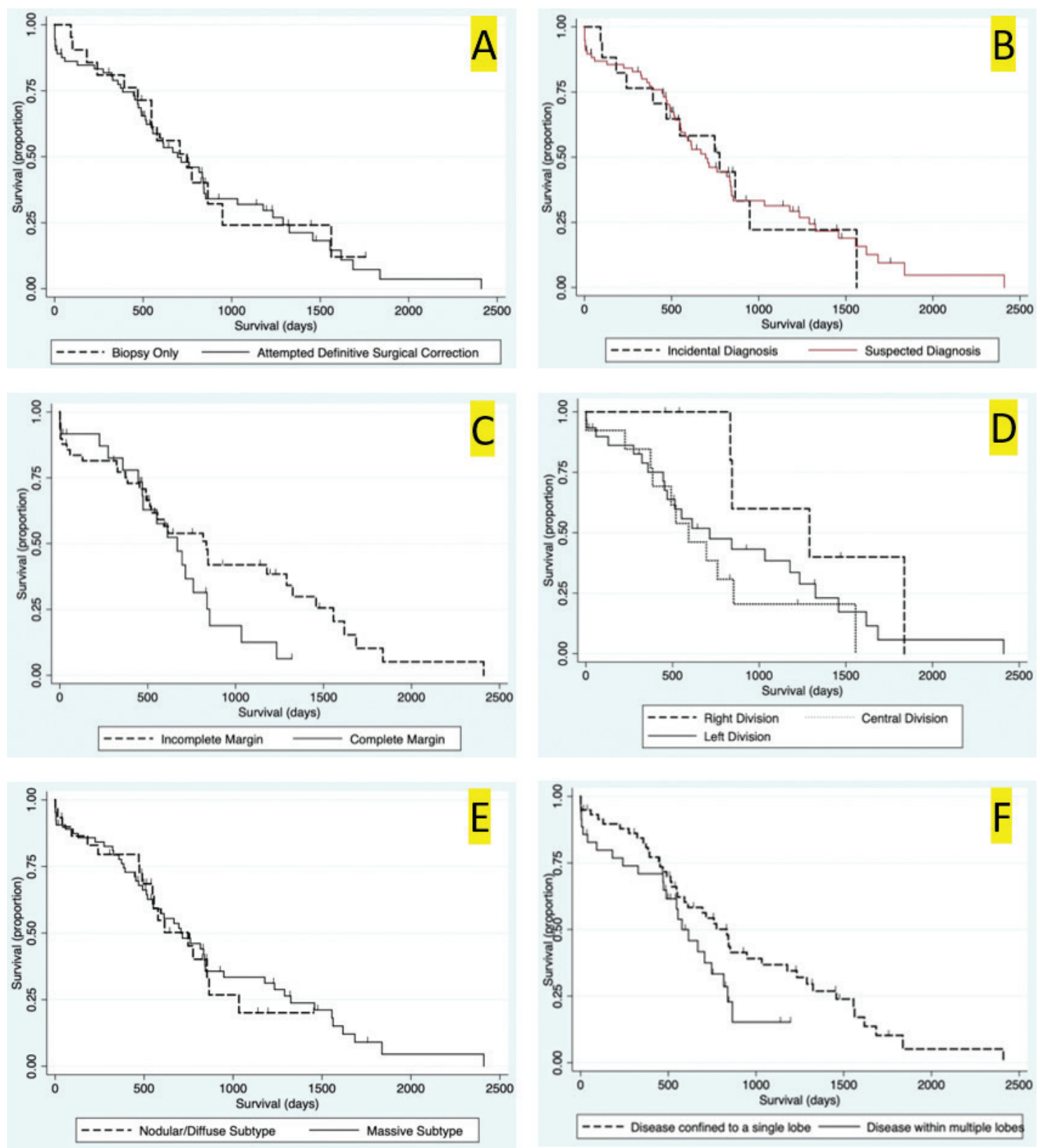

Fig. 3. Kaplan-Meier survival curves for long-term outcome: (A) Kaplan-Meier survival curve demonstrating no significant difference in survival between the group that had definitive surgical resection and those that had only had a biopsy carried out $(\mathrm{HR}=1.07, \mathrm{CI}=0.60-1.91, p=0.810)$. (B) Kaplan-Meier survival curve demonstrating that there was no significant difference in survival between those dogs incidentally diagnosed via a surgical biopsy during abdominal exploratory surgery for another disease process and dogs with presumptive preoperative diagnosis of HCC [ dogs with attempted surgical resection of a liver mass $(n=$ $73)$ or biopsy of a mass that was deemed unresectable $(n=4)](\mathrm{HR}=0.98, \mathrm{CI}=0.52-1.84, p=0.943)$. (C) Kaplan-Meier survival curve of dogs undergoing attempted definitive surgical resection of HCC. There was no significant difference in survival between dogs that had complete versus incomplete surgical margins $(\mathrm{HR}=1.63, \mathrm{CI}=0.89-2.98, p=0.111)$. (D) Kaplan-Meier survival curve of dogs with HCC confined to a single liver lobe undergoing attempted definitive surgical resection. No significant difference in survival was identified between dogs with tumors in the right, central or left division. Right versus central $(\mathrm{HR}=2.98, \mathrm{CI}=$ $0.92-9.63, p=0.068)$, right versus left $(\mathrm{HR}=2.09, \mathrm{CI}=0.72-6.11, p=0.177)$, and central versus left $(\mathrm{HR}=0.70, \mathrm{CI}=0.33-1.47$, $p=0.349$ ). (E) Kaplan-Meier survival curve demonstrating no significant difference in survival in dogs with massive subtype and those without a massive subtype (diffuse or nodular) $(\mathrm{HR}=0.92, \mathrm{CI}=0.53-1.61, p=0.773)$. (F) Kaplan-Meier survival curve demonstrating no significant difference in survival in dogs with HCC confined to a single liver lobe and those with HCC affecting multiple lobes $(\mathrm{HR}=1.65, \mathrm{CI}=0.96-2.83, p=0.070)$. 
Table 1. Variables associated with overall survival in all 94 patients with a histopathologic diagnosis of HCC following multivariable Cox regression analysis.

\begin{tabular}{lccc}
\hline Variable & Hazard Ratio & $\mathbf{9 5 \%}$ CI & p-value \\
\hline HCC of quadrate lobe & 2.09 & $1.24-3.53$ & 0.006 \\
Elevated K (per unit mEq/L) & 1.81 & $1.14-2.87$ & 0.011 \\
Elevated BUN (per unit mg/dL) & 1.02 & $1.01-1.04$ & 0.002 \\
Elevated GGT (per unit U/L) & 1.00 & $1.00-1.00$ & 0.025 \\
Age (in months) & 1.01 & $1.01-1.02$ & 0.004 \\
\hline
\end{tabular}

Table 2. Variables associated with overall survival in those 73 patients that underwent surgical resection for definitive treatment of HCC following multivariable Cox regression analysis.

\begin{tabular}{lccc}
\hline Variable & Hazard ratio & $\mathbf{9 5 \%}$ CI & p-value \\
\hline $\mathrm{ASA} \geq 4$ & 2.50 & $1.02-6.16$ & 0.046 \\
HCC of the quadrate lobe & 3.22 & $1.47-6.64$ & 0.006 \\
Elevated K (per unit mEq/l) & 2.24 & $1.27-3.95$ & 0.006 \\
Elevated BUN (per unit mg/dl) & 1.03 & $1.01-1.04$ & 0.002 \\
Elevated GGT (per unit U/l) & 1.00 & $1.00-1.00$ & 0.022 \\
\hline
\end{tabular}

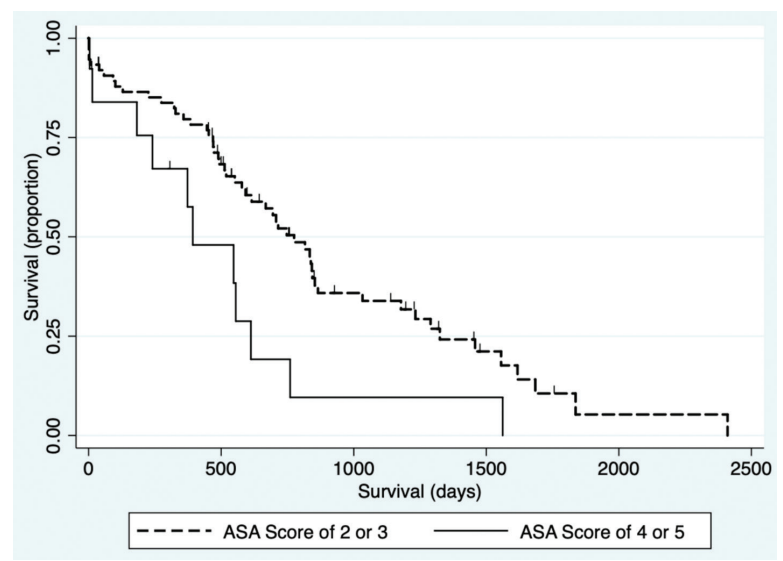

Fig. 4. Kaplan-Meier survival curve demonstrating that dogs with an ASA score of 4 or 5 prior to surgery had a significant association with a decreased survival in all patients $(n=94)$ in the final multivariable model $(\mathrm{HR}=2.94, \mathrm{CI}=1.43-6.01$, $p=0.003)$.

differences, HCC can still be considered to have a good prognosis following diagnosis.

Contrary to our hypothesis, this study failed to demonstrate a significant difference in MST between those dogs that had an attempted definitive surgery to remove the tumor, and those that only had a biopsy carried out for either non-resectable tumors, or incidentally found liver pathology (Fig. 3a). Interestingly, one of the dogs that had a tumor biopsied after it was deemed unresectable at the time of surgery by the operative surgeon was still alive at the conclusion of this study, more than 1,700 days later. The failure of finding a survival advantage in dogs undergoing

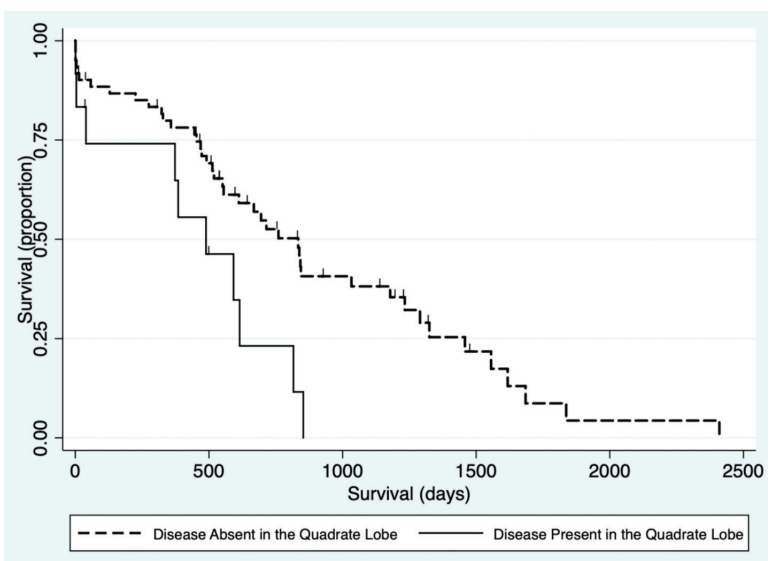

Fig. 5. Kaplan-Meier survival curve demonstrating that HCC present in the quadrate lobe had a significant association with a decreased survival in patients undergoing attempted definitive surgical resection $(n=73)$ in the final multivariable model $(\mathrm{HR}=3.11, \mathrm{CI}=1.47-6.59, p=0.003)$.

attempted surgical resection of their tumors should be interpreted with caution. This study recruited cases from a histopathological data bank, meaning that all dogs in this cohort either had tumors that were diagnosed preoperatively and surgical resection appeared feasible, or had liver abnormalities found during exploratory celiotomy prompting biopsy. This data set does not include dogs with HCC where surgery was declined either due to clinical opinion that the particular case may not benefit from surgery or those dogs where surgery was declined by owners for a multitude of reasons. We are, therefore, still unable to accurately assess the effect of attempted definitive surgery on survival. Such analysis 
would require the comparison of outcome in dogs with equivalent disease following randomized treatment. Additionally, this cohort included a larger number of dogs that were incidentally diagnosed with HCC than reported previously (Liptak et al., 2004; Linden et al., 2019). The reason for this finding compared to previous evaluations is unknown; however, the lack of influence of attempted surgical resection on long-term survival highlights that HCC is unlikely to progress as a systemic disease and significant clinical signs may be secondary to its local presence.

The findings of this study failed to demonstrate any significant difference in MST with completeness of surgical margins obtained during attempted surgical resection (Fig. 3c). This agreed with our hypothesis and likely due to the low reported metastatic rate of HCC. Previous reports on the association of complete resection and MST have been conflicting (Liptak et al., 2004; Matsuyama et al., 2017). One possible explanation is that histopathology does not allow the evaluation of the entire surgical margin and is only an estimate of actual margins. This could confound the results of retrospective studies such as this one. Despite this, survival times are still likely to be prolonged in patients that have their tumors incompletely resected. The perioperative mortality rate in this study was $6.4 \%$, which is similar to previous studies of surgery for patients undergoing liver lobectomy (Liptak et al., 2004; Hanson et al., 2017; Linden et al., 2019). An increased risk of death before discharge was found in dogs with HCC in the right middle liver lobe. Isolation and resection of the right and central division of the liver is technically more difficult due to exposure and vascular anatomy (Liptak et al., 2004; Linden et al., 2019). A 40\% intraoperative mortality rate has been reported for patients with right-sided liver tumors secondary to intractable hemorrhage (Liptak et al., 2004). Similarly, the incidence of perioperative hemorrhage in dogs having surgery to remove lobes in the central division is reportedly 33\% (Linden et al., 2019). While the need for blood transfusion has been associated with decreased survival to discharge in patients undergoing hepatic surgery (Hanson et al., 2017); this was not repeatable in our study. The transfusion rate in this study was approximately $9 \%$, which is similar to a previous report (Hanson et al., 2017), with no predilection for localization of disease. Although patients with disease present within the right medial liver lobe were at higher odds of dying prior to discharge in this cohort, this association was lost when considering long-term survival which is similar to a previous report (Liptak et al., 2004). Due to the increased risk of removing the central division of the liver (Liptak et al., 2004; Linden et al., 2019), and the long survival times that can be achieved without completely removing the tumor, surgical debulking of $\mathrm{HCC}$ in the central division may be considered in those instances when a more aggressive surgery is likely to put the patient's life at risk.

In this study, no difference was found in MST in dogs with disease identified in multiple versus single lobes (Fig. 3f) or between morphological subtypes (Fig. 3e). Historically, nodular or diffuse HCC subtypes have been thought to have a worse prognosis compared to massive HCC (Liptak and Withrow, 2013). This could be secondary to the surgical amenability of the massive subtype or presumably the increased liver function compromise that may occur with the nodular and diffuse forms (Liptak and Withrow, 2013). The difference found in this study could be due inconsistencies in identification of subtype, or reporting by the operative surgeon when compared to the original description by Patnaik et al. (1980). However, the incidence of the massive morphological subtype in this study was very similar to that in the afore-mentioned original study (69\% vs. 61\%, respectively) (Patnaik et al., 1980).

Multivariable Cox regression in this study revealed presence of disease within the quadrate lobe, elevated potassium, elevated BUN value, and elevated GGT as negative prognostic indicators for survival for all patients. Older patients had shorter survival times when evaluating all patients, and patients with ASA scores of 4 and 5 had shorter survival times for patients undergoing attempted definitive surgical removal (Tables 1 and 2, respectively). Both elevated BUN and GGT have been historically identified as risk factors for death in dogs undergoing biliary surgery (Amsellem et al., 2006). Elevated GGT has been implicated as a poor prognostic indicator in dogs undergoing treatment for septic peritonitis and open peritoneal drainage (Winkler and Greenfield, 2000), and has also been used as a prognostic indicator in the human literature (KazemiShirazi et al., 2007). In addition, hepatomas and HCC may be functional in secreting tumor-specific GGT and thus elevated GGT levels may be associated with a higher likelihood of metastases (Yao et al., 2000). Potassium values have been shown to be elevated in end stage or terminal liver disease in people and, therefore, may reflect dogs with more severe disease (Ahya et al., 2006). The ASA score is discernably a reflection the effect of the patient's overall systemic health. ASA score has been demonstrated to be a prognostic indicator and predictor for the likelihood of postoperative infection, complications, and survival time in humans and dogs in previous studies (Eugster et al., 2004; Woodfield et al., 2007; Djaladat et al., 2014). Although the association of shortened survival and disease in the right medial liver lobe were lost when carrying out multivariable Cox regression, a negative association was found between presence of disease within the quadrate lobe and long-term survival. Therefore, identification of disease present within the central division preoperatively can serve as an overall negative prognostic indicator to the owner. 
The secondary objective of this study was to evaluate the usefulness of preoperative diagnostics for intraoperative planning. Although cytology returned a diagnosis of $\mathrm{HCC}$ in $46.9 \%$ of the cases, portentously, it was inconclusive or suggestive of a non-malignant process in $40.6 \%$ of the cases. Therefore, the results of this study suggest that a large percentage of HCC cannot be diagnosed on cytology alone. As non-malignant and inconclusive diagnostic results were possible, it is prudent to continue to assess the entire clinical picture when assessing dogs with probable HCC. The lack of agreement between cytology and wedge biopsy has been noted previously with only $52 \%$ agreement (Cole et al., 2002; Bahr et al., 2013). Given these findings, FNA must be interpreted with caution in the preoperative work-up of patients with suspected HCC.

The agreement of the extent and localization of disease to the left, central, and right divisions between ultrasound and surgery, CT, and surgery, and ultrasound and CT were very high. Previous studies have shown similar accuracy for both CT and ultrasound (Liptak et al., 2004). Preoperative localization of the tumor is important for surgical planning and to guide owner expectations. The accuracy of these tests is likely partially due to the skill of the radiologist, and the low rate of metastasis of HCC. CT was not superior to ultrasound in contrast to our hypothesis in this study. CT may remain superior for differentiation between the underlying pathology of identified hepatic masses (Jang et al., 2009; Kutara et al., 2014); however, contrast and harmonic ultrasonography may hold promise in this application (Kutara et al., 2006; Fukushima et al., 2012). Overall, these findings suggest that the use of either imaging modality serves for excellent preoperative localization of disease.

The present study had numerous limitations. Being a retrospective study, not all patients underwent a standardized treatment regimen and complete medical records were not available for all patients. For example, although elevated ALT was not identified as a prognostic factor in this study, patients with incidentally diagnosed HCC had lower ALT levels that may have influenced the analysis of the impact of attempted definitive surgery on survival. In addition, patients were not euthanized at a standard point in disease progression, rather it varied, likely due to the difference in perceived quality of life by owners; ultimately altering MST. Our method of case selection is based on the histopathologic diagnosis introduced sample bias, likely resulting in missing clinical cases of HCC that never had surgery; however, these clinical cases are underrepresented in the literature as a whole. There were many pathologists and surgeons involved in the assessment of these cases which may have influenced our results. Additionally, there was no standardized indication for surgery and patients had varying severity of clinical signs before surgery. A prospective study would be required to definitively evaluate the impact of surgery and histopathological subtype on survival.

Patients who are diagnosed with HCC carry a good long-term prognosis. The primary systemic effect of HCC appears to be its local presence, causing spaceoccupying issues, hepatic dysfunction secondary to local invasion, or spontaneous rupture leading to hemoabdomen. Although surgical intervention can alleviate clinical signs related to the local presence of the disease, surgical intervention in itself may not improve prognosis given the low systemic progression of canine HCC. In conclusion, when considering the results of this and previous studies, the primary role of surgery in the treatment of HCC should focus on local tumor control and be tempered in light of the risk to the patient. Dogs with HCC can enjoy prolonged survival times even if clean surgical margins are not obtained.

\section{Acknowledgements}

The authors would like to thank Mr. Steven Parry from the Cornell university statistical consulting unit for his assistance with this manuscript.

\section{Conflict of interest}

The authors declare that there is no conflict of interest Authors' contribution

All authors contributed to the study design. All authors read and approved the final manuscript.

References
Ahya, S.N., Soler, M.J., Levitsky, J. and Batlle D. 2006. Acid-base and potassium disorders in liver disease. Semin. Nephrol. 26(6), 466-470.

Amsellem, P.M., Seim, H.B., MacPhail, C.M., Bright, R.M., Twedt, D.C., Wrigley, R.H. and Monnet, E. 2006. Long-term survival and risk factors associated with biliary surgery in dogs: 34 cases (1994-2004). J. Am. Vet. Med. 229, 1451-1457.

Bahr, K.L., Sharkey, L.C., Murakami, T. and Feeney, D.A. 2013. Accuracy of US-guided FNA of focal liver lesions in dogs: 140 cases (2005-2008). J. Am. Anim. Hosp. Assoc. 49, 190-196.

Balkman, C. 2009. Hepatobiliary neoplasia in dogs and cats. Vet. Clin. Small Anim. Pract. 39, 617-625.

Cole, T.1., Center, S.A., Flood, S.N., Rowland, P.H., Valentine, B.A., Warner, K.L. and Hollis, N.E. 2002. Diagnostic comparison of needle and wedge biopsy specimens of the liver in dogs and cats. J. Am. Vet. Med. Assoc. 220, 1483-1490.

Djaladat, J., Bruins, H.M., Miranda, G., Cai, J., Skinner, E.C. and Daneshmand, S. 2014. The association of preoperative serum albumin level and American Society of Anesthesiologists (ASA) score on early complications and survival of patients undergoing radical cystectomy for a urothelial bladder cancer. BJU Int. 113, 887-893.

Eugster, S., Schawalder, P., Gaschen, F. and Boelin, P. 2004. A prospective study of postoperative surgical 
site infection in dogs and cats. Vet. Surg. 33, 542550.

Fukushima, K., Kanemoto, H., Ohno, K., Takahashi, M., Nakashima, K., Fujino, Y., Uchida, K., Fujiwara, R., Nishimura, R. and Tsujimoto, H. 2012. CT characteristics of primary hepatic mass lesions in dogs. Vet. Radiol. Ultrasound 53, 252-257.

Hanson, K.R., Pigott, A.M. and Linklater, A.K. 2017. Incidence of blood transfusion requirement and factors associated with transfusion following liver lobectomy in dogs and cats: 72 cases (2007-2015). J. Am. Vet. Med. Assoc. 251, 929-934.

Jang, H.J., Yu, H. and Kim, T.K. 2009. Contrastenhanced ultrasound in the detection and characterization of liver tumors. Cancer Imaging 9, 96-103.

Kazemi-Shirazi, L., Endler, G., Winkler, S., Schickbauer, T., Wagner, O. and Marsik, C. 2007. Gamma glutamyltransferase and long-term survival: is it just the liver? Clin. Chem. 53, 940946.

Kinsey, J.R., Gilson, S.D., Hauptman, J., Mehler, S.J., and May, L.R. 2015. Factors associated with longterm survival in dogs undergoing liver lobectomy as treatment for liver tumors. Can. Vet. J. 56, 598-604.

Kosovsky, J.E. 1989. Results of partial hepatectomy in 18 dogs with hepatocellular carcinoma. J. Am. Anim. Hosp. Assoc. 25, 203-206.

Kutara, K., Asano, K., Kito, A., Teshima, K., Kato, Y., Sasaki, Y., Edamura, K., Shibuya, H., Sato, T., Hasegawa, A. and Tanaka,S. 2006. Contrast harmonic imaging of canine hepatic tumors. J. Vet. Med. Sci. 68, 433-438.

Kutara, K., Seki, M., Ishikawa, C., Sakai, M., Kagawa, Y., Iida, G., Ishigaki, K., Teshima, K., Edamura, K., Nakayama, T. and Asano, K. 2014. Triple-phase helical computed tomography in dogs with hepatic masses. Vet. Radiol. Ultrasound 55, 7-15.

Linden, D.S., Liptak, J.M., Vinayak, A., Cappelle, K., Hoffman, C., Fan, S., Smiley, W. and Matz, B.M. 2019. Outcomes and prognostic variables associated with central division hepatic lobectomies: $61 \mathrm{dogs}$. Vet. Surg. 48, 309-314.
Liptak, J.M. and Withrow, S.J. 2013. Hepatobiliary tumors. In: Small animal clinical oncology, 5th ed. Eds., Withrow, S.J., Vail, D.M. and Page, R.L. Philadelphia, PA: Elsevier Sauders, pp: 405-412.

Liptak, J.M., Dernell, W.S., Monnet, E., Powers, B.E., Bachand, A.M., Kenney, Juanita, G. and Withrow, S.J. 2004. Massive hepatocellular carcinoma in dogs: 48 cases (1992-2002). J. Am. Vet. Med. Assoc. 225, 1225-1230.

Matsuyama, A., Takagi, S., Hosoya, K., Kagawa, Y., Nakamura, K., Deguchi, T. and Takiguchi, M. Impact of surgical margins on survival of $37 \mathrm{dogs}$ with massive hepatocellular carcinoma. 2017. N Z Vet. J. 65, 227-231.

Patnaik, A.K., Hurvitz, A.I. and Lieberman, P.H. 1980. Canine hepatic neoplasms: a clinicopathologic study. Vet. Pathol. 17, 553-564.

Patnaik, A.K., Hurvitz, A.I., Lieberman, P.H. and Johnson, G.F. 1981. Canine hepatocellular carcinoma. Vet. Pathol. 18, 427-438.

Sleight, D.R. and Thomford, N.R. 1970. Gross anatomy of the blood supply and biliary drainage of the canine liver. Anat. Rec. 166, 153-160.

Van Sprundel, R.G.H.M., van den Ingh, T.S.G.A.M. and Guscetti, F., Kershaw, O., Kanemoto, H, van Gils, H.M., Rothuizen, J., Roskams, T. and Spree, B. 2013. Classification of primary hepatic tumours in the dog. Vet. J. 197, 596-606.

Winkler, K.P. and Greenfield, C.L. 2000. Potential prognostic indicators in diffuse peritonitis treated with open peritoneal drainage in the canine patient. J. Vet. Emerg. Crit. Care 10, 259-265.

Woodfield, J.C., Beshay, N.M.Y., Pettigrew, R.A., Plank, L.D. and van Rij, A.M. 2007. American society of anesthesiologists classification of physical status as a predictor of wound infection. ANZ J. Surg. 777, 738-741.

Yao, D., Jiang, D., Huang, Z., Lu, J., Tao, Q., Yu, Z. and Meng, X. 2000. Abnormal expression of hepatoma specific $\gamma$-glutamyl transferase and alteration of $\gamma$-glutamyl transferase gene methylation status in patients with hepatocellular carcinoma. Cancer 88, 761-769. 